\title{
Fixed Points of Monotone Total Asymptotically Nonexpansive Mapping in Hyperbolic Space via New Algorithm
}

\author{
Amna Kalsoom, ${ }^{1}$ Maliha Rashid, ${ }^{1}$ Tian-Chuan Sun $\mathbb{D}^{2},{ }^{2}$ Amna Bibi, ${ }^{3}$ Abdul Ghaffar $\left(\mathbb{D},{ }^{4}\right.$ \\ Mustafa Inc $\mathbb{D},{ }^{5,6,7}$ and Ayman A. Aly \\ ${ }^{1}$ Department of Mathematics and Statistics, International Islamic University, Islamabad, Pakistan \\ ${ }^{2}$ Qiuzhen College, Huzhou University, Huzhou 313000, China \\ ${ }^{3}$ School of Mathematics and Information Science, Henan Polytechnic University, Jiaozuo 454000, China \\ ${ }^{4}$ Department of Mathematics, Ghazi University, D G Khan, Pakistan \\ ${ }^{5}$ Department of Computer Engineering, Biruni University, Istanbul, Turkey \\ ${ }^{6}$ Department of Mathematics, Science Faculty, Firat University, 23119 Elazig, Turkey \\ ${ }^{7}$ Department of Medical Research, China Medical University Hospital, China Medical University, Taichung, Taiwan \\ ${ }^{8}$ Department of Mechanical Engineering, College of Engineering, Taif University, P.O. Box 11099, Taif 21944, Saudi Arabia
}

Correspondence should be addressed to Tian-Chuan Sun; suntch@zjhu.edu.cn and Mustafa Inc; minc@firat.edu.tr

Received 11 May 2021; Accepted 7 July 2021; Published 29 July 2021

Academic Editor: Nawab Hussain

Copyright ( 2021 Amna Kalsoom et al. This is an open access article distributed under the Creative Commons Attribution License, which permits unrestricted use, distribution, and reproduction in any medium, provided the original work is properly cited.

In this article, we consider an extensive class of monotone nonexpansive mappings and introduce a new iteration algorithm to approximate the fixed point for monotone total asymptotically nonexpansive mappings in the framework of hyperbolic space. Faster convergence and stability results are proved for that iteration; also, fixed point is approximated numerically in a nontrivial example by using MATLAB.

\section{Introduction}

The concept of hyperbolic space was given by Reich and Shafrir [1] in 1990, which is defined as a metric space $(\Sigma, \sigma)$ that has a family Fourier of metric lines; for any two unique endpoints $l, m \in \Sigma$, there is a unique metric line in Fourier. This metric line works out a unique metric segment symbolize by $[l, m]$, which is an isometric image of $[0, \sigma(l, m)]$. A unique point $z \in[l . m]$ is denoted by $\alpha l \oplus(1-\alpha) m$ which satisfies

$$
\begin{gathered}
\sigma(l, z)=(1-\alpha) \sigma(l, m), \\
\sigma(z, m)=\alpha \sigma(l, m),
\end{gathered}
$$

where $\alpha \in[0,1]$. Such a metric space is called a convex metric space, such that

$$
\sigma(\alpha l \oplus(1-\alpha) m, \alpha p \oplus(1-\alpha) q) \leq \alpha \sigma(l, p)+(1-\alpha) \sigma(m, q)
$$

for all $l, m, p, q \in \Sigma$, then $\Sigma$ is called hyperbolic metric space (abbreviated as H.M.S).

The class of hyperbolic spaces contains the normed spaces, CAT(0) spaces, and many others. There are many examples in literature which show that hyperbolic spaces are more general than Banach spaces; for details, see [2] and Example 1.1 of [3].

Recently, a new direction has been discovered dealing with the extension of the Banach Contraction Principle [4] to partially ordered metric spaces. The case of monotone nonexpansive mappings was first considered in [5]. After that, Dehaish and Khamsi [6] gave an analogue to Browder [7] and Göhde [8] fixed point theorems for monotone nonexpansive mappings. In 2018, Alfuraidan and Khamsi [9] extended Goebel and Kirk's fixed point theorem [10] for 
asymptotically nonexpansive mappings to the case of monotone mappings. Multiple articles [11-14] can be found in the literature on fixed point of asymptotically nonexpansive mapping using multistep iterations and strong convergence analysis.

In 2016, Alber et al. [15] introduced the concept of total asymptotically nonexpansive mappings that generalizes the family of mapping that are the extension of asymptotically nonexpansive mappings. Example 1 of [16] and Example 3.1 of [4] show that total asymptotically nonexpansive mappings properly contain the asymptotically nonexpansive mappings.

In this article, we define monotone total asymptotically nonexpansive mappings (abbreviated as M.T.A.N.M) and also extend Alber's fixed point theorem [10] for the respective class. In addition, this result also generalizes the results of Alfuraidan and Khamsi in hyperbolic space [9].

In Section 4, we introduce a new iteration scheme, prove fast convergence and stability results, and also provide comparison with some famous iterations listed as Banach [17], Mann [18], Ishikawa [19], Agarwal et al. [20], Noor [21], Abbas and Nazir [22], Vatan Two-step [23], an accelerated iteration [24] (by Chen and Wen [24]), and Thakur New [25]. Numerically, we compare the convergence of new iteration with these iterations in a nontrivial example.

\section{Preliminaries}

Let $(\Sigma, \sigma, \preceq)$ be a partially ordered (abbreviated as P.O) metric space, any two points $a, b \in \Sigma$ are comparable whenever $a \leq b$ or $b \preceq a$.

Definition 1 . Consider $(\Sigma, \sigma, \preceq)$ be a partially ordered space and $Y$ be a self map of $\Sigma$, which is said to be

(i) monotone or order preserving [5] if

$$
a \preceq b \Rightarrow Y a \preceq Y b
$$

(ii) monotone Lipschitzian mapping [5] if $Y$ is order preserving and there exist $L \geq 0$ such that

$$
\sigma(Y(a), Y(b)) \leq H \sigma(a, b)
$$

If $H=1$, the mapping $Y$ is said to be order preserving nonexpansive mapping

(iii) monotone asymptotically nonexpansive mapping [9] if there exists a sequence $\left\{H_{v}\right\}$ for $v \in \mathbb{N}$ such that

$$
\begin{gathered}
\lim _{v \longrightarrow \infty} H_{v}=1, \\
\sigma\left(Y^{v}(a), Y^{v}(b)\right) \leq H_{v} \sigma(a, b),
\end{gathered}
$$

for every $a, b \in \Sigma$, such that $a$ and $b$ are comparable

Now, we will define M.T.A.N.M in hyperbolic space.

Definition 2. Let $\Sigma$ be a hyperbolic metric space having a nonempty subset $K$. A self map $Y$ is monotone total asymptotically nonexpansive mapping on $K$ if there exist nonnegative sequences $\left\{\mu_{v}\right\}$ and $\left\{\xi_{v}\right\}$ with $\mu_{v} \longrightarrow 0, \xi_{v} \longrightarrow 0$, as $v \longrightarrow$ $\infty$, a strictly increasing continuous function

$$
\vartheta:[0, \infty) \longrightarrow[0, \infty) \text { with } \vartheta(0)=0,
$$

such that

$$
\sigma\left(Y^{v} s, Y^{v} t\right) \leq \sigma(s, t)+\mu_{v} \vartheta(\sigma(s, t))+\xi_{v} \text { for all } v \geq 1
$$

and there exists a constant $R^{*}>0$ such that $\vartheta(\lambda) \leq R^{*} \lambda$ for $\lambda>0$, then

$$
\sigma\left(Y^{v} s, Y^{v} t\right) \leq\left(1+R^{*} \mu_{v}\right) \sigma(s, t)+\xi_{v},
$$

for every comparable elements $s, t \in K$.

Example 1. Consider the real line $\mathbb{R}$ as a hyperbolic metric space and $K$ be the subset of $\mathbb{R}, K=[0, \pi / 2]$, and $T: K \longrightarrow$ $K$ be a mapping defined by $T x=\sin x$. Suppose that there exist two nonnegative sequences $\left\{x_{n}\right\}$ and $\left\{\xi_{n}\right\}$ with $x_{n} \longrightarrow 0$ and $\xi_{n} \longrightarrow 0$ as $n \longrightarrow 0$ and a strictly increasing continuous function $\theta:[0, \infty) \longrightarrow[0, \infty)$ with $\theta(0)=0, \theta$ $(\lambda)=\lambda+1, \lambda \in[0, \infty)$. Then, T is M.T.A.N.M.

Example 2. Consider the real plane $\mathbb{R}^{2}$ as a hyperbolic metric space. Let $\sigma: \Sigma \times \Sigma \longrightarrow \mathbb{R}$ be defined as

$$
\sigma(w, h)=\left|w_{1}-h_{1}\right|+\left|w_{1} w_{2}-h_{1} h_{2}\right| \text { where } w=\left(w_{1}, w_{2}\right), h=\left(h_{1}, h_{2}\right) \text {. }
$$

Let $K=[0,1] \times[0,1] \subset \Sigma$ and $Y: K \longrightarrow K$ be a mapping defined by

$$
Y(w, h)= \begin{cases}\left(\frac{w}{10}, \frac{h}{10}\right) & \text { if }(w, h) \neq\left(\frac{1}{2}, \frac{1}{2}\right) \\ (0,0) & \text { if }(w, h)=\left(\frac{1}{2}, \frac{1}{2}\right) .\end{cases}
$$

Thus, $(0,0)$ is the fixed point of $Y$. Suppose $\exists$ two nonnegative sequences $\left\{\mu_{v}\right\}$ and $\left\{\xi_{v}\right\}$ with $\mu_{v} \longrightarrow 0, \xi_{v} \longrightarrow 0$, as $v \longrightarrow \infty$, and a strictly increasing continuous function

$$
\vartheta:[0, \infty) \longrightarrow[0, \infty) \text { with } \vartheta(0)=0
$$

Now, we consider the following cases with assumptions $\left(w_{1}, w_{2}\right) \preceq\left(h_{1}, h_{2}\right)$ : 
Case 1. If $\left(w_{1}, w_{2}\right)=(1 / 2,1 / 2)=\left(h_{1}, h_{2}\right)$, then $Y\left(w_{1}, w_{2}\right)=$ $(0,0)=Y\left(h_{1}, h_{2}\right)$. In this case, $Y$ is monotone and satisfies all the conditions of total asymptotically nonexpansive mapping.

Case 2. If $\left(w_{1}, w_{2}\right)=(1 / 2,1 / 2),\left(h_{1}, h_{2}\right) \neq(1 / 2,1 / 2)$, then $Y$ $\left(w_{1}, w_{2}\right)=(0,0), Y\left(h_{1}, h_{2}\right)=\left(h_{1} / 10, h_{2} / 10\right)$, and $Y^{v}\left(w_{1}, w_{2}\right)$ $=(0,0), Y^{v}\left(h_{1}, h_{2}\right)=\left(h_{1} / 10^{v}, h_{2} / 10^{v}\right)$. Also,

$$
\begin{aligned}
& \sigma\left(Y^{v}\left(w_{1}, w_{2}\right), Y^{v}\left(h_{1}, h_{2}\right)\right)=\frac{h_{1}}{10^{v}}+\frac{h_{1} h_{2}}{10^{2 v}} \leq\left|\frac{1}{2}-h_{1}\right| \\
& +\left|\frac{1}{2^{2}}-h_{1} h_{2}\right| \leq \sigma\left(\left(w_{1}, w_{2}\right),\left(h_{1}, h_{2}\right)\right) \\
& \quad+\mu_{v} \vartheta\left(\sigma\left(\left(w_{1}, w_{2}\right),\left(h_{1}, h_{2}\right)\right)\right)+\xi_{v}
\end{aligned}
$$

implies that $Y$ is a M.T.A.N.M.

Case 3. If $\left(w_{1}, w_{2}\right) \neq(1 / 2,1 / 2) \neq\left(h_{1}, h_{2}\right)$, then $Y\left(w_{1}, w_{2}\right)=$ $\left(w_{1} / 10, w_{2} / 10\right), Y\left(h_{1}, h_{2}\right)=\left(h_{1} / 10, h_{2} / 10\right)$, and $Y^{v}\left(w_{1}, w_{2}\right)$ $=\left(w_{1} / 10^{v}, w_{2} / 10^{v}\right), Y^{v}\left(h_{1}, h_{2}\right)=\left(h_{1} / 10^{v}, h_{2} / 10^{v}\right)$. Now,

$$
\begin{aligned}
& \sigma\left(Y^{v}\left(w_{1}, w_{2}\right), Y^{v}\left(h_{1}, h_{2}\right)\right)=\left|\frac{w_{1}-h_{1}}{10^{v}}\right|+\left|\frac{w_{1} w_{2}-h_{1} h_{2}}{10^{2 v}}\right| \\
& \quad \leq\left|w_{1}-h_{1}\right|+\left|w_{1} w_{2}-h_{1} h_{2}\right| \leq \sigma\left(\left(w_{1}, w_{2}\right),\left(h_{1}, h_{2}\right)\right) \\
& \quad+\mu_{v} \vartheta\left(\sigma\left(\left(w_{1}, w_{2}\right),\left(h_{1}, h_{2}\right)\right)\right)+\xi_{v} .
\end{aligned}
$$

Hence, $Y$ is M.T.A.N.M.

Next, we have some definitions and lemmas that will be useful in the proof of the main result.

Definition 3 (see [26]). A hyperbolic space $\Sigma$ with metric $\sigma$ is said to be uniformly convex if for any $w \in \Sigma$, for every $z>0$, and for each $\varepsilon>0$

$$
\begin{gathered}
\delta(z, \varepsilon)=\inf \left\{1-\frac{1}{z} \sigma\left(\frac{1}{2} s \oplus \frac{1}{2} t, w\right) ; \sigma(s, w)\right. \\
\leq z, \sigma(t, w) \leq z, \sigma(s, t) \geq z \varepsilon\}>0 .
\end{gathered}
$$

The function $\delta$ is called the modulus of uniform convexity of $\Sigma$.

A hyperbolic space $(\Sigma, \sigma)$ satisfies the property $(R)[26]$. If $\left\{K_{v}\right\}$ is nonincreasing sequence of nonempty, bounded, closed, and convex subset of $\Sigma, \cap \underset{v=1}{\infty} K_{v} \neq \vartheta$.

Definition 4 (see [27]). A bounded sequence $\left\{r_{v}\right\} \in \Sigma$ is $\Delta$ -convege to $r \in \Sigma$, if $r$ is the unique asymptotic centre of every subsequence $\left\{r_{v k}\right\}$ of $\left\{r_{v}\right\}$.

Definition 5 (see [9]). A partially ordered hyperbolic metric space $\Sigma$ satisfies the monotone weak Opial condition if any sequence in $\Sigma$ which is monotone and weakly converges to $s$, then the following

$$
\underset{v \longrightarrow \infty}{\limsup } \sigma\left(s_{v}, s\right) \leq \underset{v \longrightarrow \infty}{\limsup } \sigma\left(s_{v}, t\right)
$$

for every $t \in \Sigma$ such that $s \preceq t$ or $t \preceq s$.

Throughout in article, the order intervals are assumed to be closed and convex and any of the subsets

$$
\begin{aligned}
& {[s, \longrightarrow)=\{y \in \Sigma ; s \preceq y\}} \\
& (\longleftarrow, t]=\{y \in \Sigma ; y \preceq t\} .
\end{aligned}
$$

for every $s, t \in \Sigma$.

Lemma 6 [9]. Suppose $(\Sigma, \sigma)$ be uniformly convex H.M.S, and $K$ be a subset of $\Sigma$ which is closed nonempty and convex. Let $\tau: K \longrightarrow[0, \infty)$ be a type function if $\exists$ a bounded sequence $\left\{s_{v}\right\} \in \Sigma$ such that

$$
\tau(s)=\underset{v \longrightarrow \infty}{\limsup _{v}} \sigma\left(s_{v}, s\right)
$$

for any $s \in K$. Since $\Sigma$ is hyperbolic space, $\tau$ is convex and continuous with distinctive minimum point $u \in K$ such that

$$
\tau(u)=\inf \{\tau(s) ; s \in K\}=\tau_{0} .
$$

Moreover, if $\left\{Y^{v}(u)\right\}$ in $K$ is the minimizing sequence of $\tau$, i.e.,

$$
\lim _{v \longrightarrow \infty} \tau\left(Y^{v}(u)\right)=\tau_{0}
$$

then, $\left\{Y^{v}(u)\right\}$ strongly converges to $u$.

\section{Main Result}

Theorem 7. Let a uniformly convex P.O H.M.S be $(\Sigma, \sigma, \preccurlyeq)$ with nonempty convex closed bounded subset $K$. Let $Y$ be a continuous M.T.A.N.M on K. Assume $\exists s_{0} \in K$, such that $s_{0} \preccurlyeq$ $Y s_{0}$. Then, $Y$ has a fixed point.

Proof. Let $s_{0} \in K$ be such that

$$
s_{0} \preccurlyeq Y s_{0} .
$$

By the monotonicity of $Y$, we get

$$
Y^{v} s_{0} \preccurlyeq Y^{v+1} s_{0}
$$

for each $v \in \mathbb{N}$, and $\left\{Y^{v} s_{0}\right\}$ is a monotone increasing sequence. Also, the order intervals are closed and convex. So, we have

$$
K_{\infty}=\bigcap_{v \geq 0}\left[Y^{v} s_{0}, \longrightarrow\right) \cap K=\cap\left\{s \in K ; Y^{v} s_{0} \preccurlyeq s\right\} \neq \phi
$$


Let $s \in K_{\infty}$, then

$$
Y^{v} s_{0} \preccurlyeq s,
$$

and the monotonicity of $Y$ implies

$$
Y\left(Y^{v} s_{0}\right)=Y^{v+1} s_{0} \leqslant Y s,
$$

for every $v \in \mathbb{N}$, i.e., $Y\left(K_{\infty}\right) \subset K_{\infty}$. Consider the type function $\tau: K_{\infty} \longrightarrow[0,+\infty)$ produced by $\left\{Y^{v} s_{0}\right\}$ given as $\tau(s)=\limsup \sigma\left(Y^{v} s_{0}, s\right)$ for any $s \in K_{\infty}$. Above lemma shows the occurrence of a unique $a \in K_{\infty}$ such that $\tau(a)$ $=\inf \left\{\tau(s) ; s \in K_{\infty}\right\}=\tau_{0}$. Since $a \in K_{\infty}$, we have $Y^{d}(a) \in$ $K_{\infty}$, for every $d \in \mathbb{N}$, which implies

$$
\begin{aligned}
\tau\left(Y^{d}(a)\right)= & \lim \sup _{v \longrightarrow \infty} \sigma\left(Y^{v} s_{0}, Y^{d} a\right) \\
& \leq \lim \sup _{v \longrightarrow \infty}\left[\sigma\left(Y^{v} s_{0}, a\right)+\mu_{p} \vartheta\left(\sigma\left(Y^{v} s_{0}, a\right)\right)+\xi_{d}\right] \\
= & \tau(a)+\mu_{d} \lim \sup _{v \longrightarrow \infty}\left(\vartheta\left(\sigma\left(Y^{v} s_{0}, a\right)\right)\right)+\xi_{d} .
\end{aligned}
$$

As $Y$ is total asymptotically nonexpansive mapping, so $\mu_{d} \longrightarrow 0, \xi_{d} \longrightarrow 0$, when $d \longrightarrow \infty$. Hence,

$$
\lim _{d \longrightarrow \infty} \tau\left(Y^{d}(a)\right)=\tau_{0}
$$

hence, $\left\{Y^{d}(a)\right\}$ is a minimizing sequence of $\tau$. Using Lemma 6, $Y^{d}(a)$ converges to $a$. Since $Y$ is continous, we have

$$
\lim _{v \longrightarrow \infty} Y\left(Y^{v} a\right)=Y(a)=a,
$$

i.e., $a$ is a fixed point of $Y$.

The following corollary is the conclusion of Theorem 3.3 of [9].

Corollary 8. Let a uniformly convex P.O H.M.S be $(\Sigma, \sigma, \preccurlyeq)$ with nonempty convex closed bounded subset $K$. Let $Y$ be a continuous M.T.A.N.M on K. Assume $\exists s_{0} \in K$, such that $s_{0} \preccurlyeq$ $Y s_{0}$. Then, $Y$ has a fixed point.

The corollary given below is the consequence of Theorem 7 by replacing the continuity condition with weak Opial condition.

Corollary 9. Let $\left(\Sigma, \sigma^{\prime}\right)$ be a uniformly convex P.O H.M.S, satisfying monotone weak Opial condition with a nonempty convex closed bounded subset K. Let $Y$ be a M.T.A.N.M on $K$. Assume $\exists s_{0} \in K$, such that $s_{0} \preccurlyeq Y s_{0}$. Then, $Y$ has a fixed point.

\section{Convergence Theorem and Stability Results}

We introduce the new iteration scheme given below, let $K$ be a nonempty convex subset of a hyperbolic space $\Sigma$, for $v \geq 0$, where $\left\{\alpha_{v}\right\},\left\{\beta_{v}\right\}$, and $\left\{\gamma_{v}\right\}$ are sequences in $[0,1]$, such that

$$
\left\{\begin{array}{l}
s_{0} \in K \\
\eta_{v}=\left(1-\gamma_{v}\right) s_{v} \oplus \gamma_{v} Y^{v} s_{v}, \\
\theta_{v}=\left(1-\beta_{v}\right) \eta_{v} \oplus \beta_{v} Y^{v} \eta_{v} \\
s_{v+1}=Y^{v}\left(\left(1-\alpha_{v}\right) Y^{v} s_{v} \oplus \alpha_{v} Y^{v} \theta_{v}\right) .
\end{array}\right.
$$

Fastness and stability play an important role for an iteration process to be preferred on another iteration process, so now, we prove that new iteration is stable and has good speed of convergence than others. For faster convergence and new class of mapping on metric space $(\Sigma, \sigma)$ introduced by Berinde [28], satisfying

$$
\sigma(Y s, Y t) \leq a \sigma(s, t)+L \sigma(s, Y s)
$$

here, we will modify this mapping as

$$
\sigma\left(Y^{v} s, Y^{v} t\right) \leq a \sigma(s, t)+L \sigma\left(s, Y^{v} s\right)
$$

for all $s, t \in \Sigma$, where $a \in[0,1)$ and $L \geq 0$.

The following definitions and lemma will be helpful for faster convergence results given in [29].

Definition 10. Let $\left\{a_{v}\right\}$ and $\left\{b_{v}\right\}$ be two sequences, having convergent points $a$ and $b$, respectively, then $\left\{a_{v}\right\}$ converges faster than $\left\{b_{v}\right\}$ if

$$
\lim _{v \longrightarrow \infty} \frac{\sigma\left(a_{v}, a\right)}{\sigma\left(b_{v}, b\right)}=0
$$

Definition 11. Let $\left\{k_{v}\right\}$ and $\left\{l_{v}\right\}$ be two fixed point schemes that converge to the same fixed point $s$. If

$$
\sigma\left(k_{v}, s\right) \leq s_{v} \text { and } \sigma\left(l_{v}, s\right) \leq t_{v} \text { for all } v \geq 0 \text {, }
$$

where $s_{v}$ and $t_{v}$ are two sequences that converge to 0 . If $s_{v}$ converges faster than $t_{v}$, then $\left\{k_{v}\right\}$ converges faster than $\left\{l_{v}\right\}$ to $s$.

Lemma 12. If $b$ is a real number such that $0 \leq b<1$ and $\left\{a_{v}\right\}_{v=0}^{\infty}$ be a sequence such that

$$
\lim _{v \longrightarrow \infty} a_{v}=0
$$

then for any positive sequence $\left\{s_{v}\right\}_{v=0}^{\infty}$ satisfying

$$
s_{v+1} \leq b s_{v}+a_{v} \Rightarrow \lim _{v \longrightarrow \infty} s_{v}=0 .
$$

Lemma 13 (see [4]). Suppose $\left\{l_{v}\right\},\left\{m_{v}\right\}$, and $\left\{\delta_{v}\right\}$ be 
sequences of nonnegative satisfying

$$
l_{v+1} \leq\left(1+\delta_{v}\right) l_{v}+m_{v} \quad \forall v \geq 1
$$

If $\Sigma \delta_{v}<\infty$ and $\Sigma m_{v}<\infty$, then $\lim _{v \longrightarrow \infty} l_{v+1}$ exists.

Lemma 14 (see [4]). Suppose $\Sigma$ be a uniformly convex H.M.S. Let $P \in[0, \infty)$ be such that

$$
\begin{gathered}
\limsup _{v \longrightarrow \infty} \sigma\left(s_{v}, a\right) \leq P, \\
\limsup _{v \longrightarrow \infty} \sigma\left(\theta_{v}, a\right) \leq P, \\
\lim _{v \longrightarrow \infty} \sigma\left(a, \alpha_{v} s_{v} \oplus\left(1-\alpha_{v}\right) \theta_{v}\right)=P,
\end{gathered}
$$

where $\alpha_{v} \in[a, b]$ with $0<a \leq b<1$. Then, we get

$$
\lim _{v \longrightarrow \infty} \sigma\left(s_{v}, \theta_{v}\right)=0
$$

Lemma 15 (see [26]). Let $\left(\Sigma, \sigma^{\prime}\right)$ be a P.O hyperbolic space. Let $K$ be a nonempty convex and closed subset of $\Sigma$. Let $Y$ $: K \longrightarrow K$ be a monotone mapping. Let $s_{1} \in K$, such that $s_{1} \preceq$ $Y s_{1}$ or $\left(Y t_{1} \preceq t_{1}\right)$. Then, the sequence $\left\{s_{v}\right\}$ in (1) then

(a) $s_{v} \preceq Y s_{v} \preceq s_{v+1}$ or $\left(s_{v+1} \preceq Y s_{v} \preceq s_{v}\right)$

(b) $s_{v} \preceq s$ or $\left(s \preceq s_{v}\right)$, provided that $\left\{s_{v}\right\} \Delta$-converge to $s \in K$, $\forall v \in \mathbb{N}$

Lemma 16. Let a uniformly convex P.O H.M.S be $(\Sigma, \sigma, \preccurlyeq)$ with nonempty convex closed bounded subset $K$. Let $Y: K$ $\longrightarrow K$ be a M.T.A.N.M with $F(Y) \neq \vartheta$. If the sequence $\left\{s_{v}\right\}$ is defined by (1) with $s_{1} \preceq Y s_{1}$ or $\left(Y s_{1} \preceq s_{1}\right)$. Then, the following holds

$$
\begin{gathered}
\text { (a) } \lim _{v \longrightarrow \infty} \sigma\left(s_{v}, s\right) \text { exist for } s \in F(Y), \\
\text { (b) } \lim _{v \longrightarrow \infty} \sigma\left(Y^{v} s_{v}, s_{v}\right)=0 .
\end{gathered}
$$

Proof. Let $s \in F(Y) \Rightarrow Y s=s$. By the above lemma $s \underline{\leq s}$, as $Y$ is monotone

$$
Y s \preceq Y s_{v} \Rightarrow s \preceq Y^{v} s_{v} .
$$

Now, using Definition 2 and after simplification, we get

$$
\sigma\left(\eta_{v}, s\right) \leq\left(1+R^{*} \mu_{v}\right) \sigma\left(s_{v}, s\right)+\xi_{v}
$$

Again using Definition 2 and (40), we get

$$
\sigma\left(\theta_{v}, s\right) \leq\left(1+R^{*} \mu_{v}\right)^{2} \sigma\left(s_{v}, s\right)+\left(2+R^{*} \mu_{v}\right) \xi_{v} .
$$

Consider

$$
\sigma\left(s_{v+1}, s\right)=\sigma\left(Y^{v}\left(\left(1-\alpha_{v}\right) Y^{v} \eta_{v} \oplus \alpha_{v} Y^{v} \theta_{v}\right), s\right)
$$

Using Definition 2, (40) and (41), we get

$$
\sigma\left(s_{v+1}, s\right) \leq\left(1+\delta_{v}\right) \sigma\left(s_{v}, s\right)+b_{v}
$$

where $\delta_{v}=\left(4+6 R^{*} \mu_{v}+4\left(R^{*} \mu_{v}\right)^{2}+\left(R^{*} \mu_{v}\right)^{3}\right) R^{*} \mu_{v}$ and $b_{v}=$ $\left(4+6 R^{*} \mu_{v}+4\left(R^{*} \mu_{v}\right)^{2}+\left(R^{*} \mu_{v}\right)^{3}\right) \xi_{v}$. Using Lemma 13, $\lim _{v \longrightarrow \infty} \sigma\left(s_{v}, s\right)$ exist for $s \in F(Y)$.

For part $(b)$, we have to show that

$$
\lim _{v \longrightarrow \infty} \sigma\left(Y^{v} s_{v}, s_{v}\right)=0
$$

the proof resembles to Theorem 2.1 of [4].

Theorem 17. Let a uniformly convex P.O H.M.S be $(\Sigma, \sigma, \preccurlyeq)$ with convex closed bounded and nonempty subset $Y$. Let $Y$ be a continuous M.T.A.N.M on $Y$, with $F(Y) \neq \vartheta$. If $\left\{s_{v}\right\}$ is defined by (1) with $s_{1} \preceq Y s_{1}$ or $\left(Y s_{1} \preceq s_{1}\right)$. If $s \preceq s_{1}$ or $\left(s_{1} \preceq s\right)$ for $s \in F(Y)$, then $\left\{s_{v}\right\} \Delta$-converges to a fixed point of $Y$.

Proof. By Lemma 16,

$$
\lim _{v \longrightarrow \infty} \sigma\left(s_{v}, s\right) \text { exist } \forall s \in F(Y)
$$

sequence is bounded and $\lim _{v \longrightarrow \infty} \sigma\left(s_{v}, Y^{v} s_{v}\right)=0$. Let $\left\{s_{v k}\right\}$ be any subsequence of $\left\{s_{v}\right\}$ for $k \in \mathbb{N}$, such that $\left\{s_{v k}\right\} \Delta$-converges to $p \in Y$. By Lemma 15, we have

$$
s_{1} \preceq s_{v k} \preceq p .
$$

Now, we have to show that every $\Delta$-convergent subsequence of $\left\{s_{v}\right\}$ has a unique $\Delta$-limit in $F(Y)$. Let $\left\{s_{v k}\right\}$ and $\left\{s_{v r}\right\}$ be two subsequences of $\left\{s_{v}\right\} \Delta$-converging to $w$ and $h$, respectively. By Lemma $16,\left\{s_{v k}\right\}$ is bounded and

$$
\lim _{v \longrightarrow \infty} \sigma\left(s_{v k}, Y^{v} s_{v k}\right)=0
$$

We claim that $w \in F(Y)$, and $\tau$ produced by $\left\{s_{v k}\right\}$ is

$$
\tau(w)=\limsup _{k \longrightarrow \infty} \sigma\left(s_{v k}, w\right) .
$$

From Theorem 7, $Y(w)=w$, same for $Y(h)=h$. By the definition of $\Delta$-convergence and Lemma 6 , we have

$$
\begin{array}{r}
\underset{k \longrightarrow \infty}{\limsup } \sigma\left(s_{v}, w\right)=\underset{k \longrightarrow \infty}{\limsup } \sigma\left(s_{v k}, w\right)<\underset{k \longrightarrow \infty}{\limsup } \sigma\left(s_{v k}, h\right) \\
=\limsup _{v \longrightarrow \infty} \sigma\left(s_{v}, h\right)=\underset{r \longrightarrow \infty}{\limsup } \sigma\left(s_{v r}, h\right) \\
<\underset{r \longrightarrow \infty}{\limsup } \sigma\left(s_{v r}, w\right)=\underset{v \longrightarrow \infty}{\limsup } \sigma\left(s_{v}, w\right),
\end{array}
$$

which is contradiction, unless $w=h$.

Theorem 18. Let a uniformly convex P.O H.M.S be $(\Sigma, \sigma, \preccurlyeq)$ with nonempty convex closed bounded subset $K$. Let $Y$ be a continuous M.T.A.N.M on $K$ with $F(Y) \neq \vartheta$. If $\left\{s_{v}\right\}$ is defined by (1) with $s_{1} \preceq Y s_{1}$ or $\left(Y s_{1} \preceq s_{1}\right)$. If $s \preceq s_{1}$ or $\left(s_{1} \preceq s\right)$ for $s \in F(Y)$, 
then $\left\{s_{v}\right\}$ converges to a fixed point of $Y$ if and only if

$$
\liminf _{v \longrightarrow \infty} \sigma\left(s_{v}, F(Y)\right)=0 \text {. }
$$

Proof. If $\left\{s_{v}\right\}$ converges to a fixed point of $Y$, then

$$
\liminf _{v \longrightarrow \infty} \sigma\left(s_{v}, F(Y)\right)=0 \quad \text { for all } v \in \mathbb{N} .
$$

Conversely, consider $\liminf _{v \longrightarrow \infty} \sigma\left(s_{v}, F(Y)\right)=0 . \quad$ From Lemma 16

$$
\lim _{v \longrightarrow \infty} \sigma\left(s_{v}, s\right) \text { exist for each } s \in F(Y)
$$

therefore, $\lim _{v \longrightarrow \infty} \sigma\left(s_{v}, F(Y)\right.$ exists. As $\liminf _{v \longrightarrow \infty} \sigma\left(s_{v}, F(Y)\right)=0$, so we get

$$
\lim _{v \longrightarrow \infty} \sigma\left(s_{v}, F(Y)\right)=0
$$

Now, we prove that $\left\{s_{v}\right\}$ is a Cauchy sequence in $K$. For $\varepsilon>0, \exists v_{0} \in \mathbb{N}$, such that for all $v \geq v_{0}$

$$
\sigma\left(s_{v}, F(Y)\right)<\frac{\varepsilon}{2}
$$

in particular inf $\left\{\sigma\left(s_{v_{0}}, s\right): s \in F(Y)\right\}<\varepsilon / 2$, so $\exists$ a fixed point $p \in F(Y)$ such that

$$
\sigma\left(s_{v_{0}}, s\right)<\frac{\varepsilon}{2}
$$

For $m, v \geq v_{0}$,

$$
\sigma\left(s_{v+m}, s_{v}\right) \leq \sigma\left(s_{v+m}, s\right)+\sigma\left(s, s_{v}\right)<2 \sigma\left(s_{v_{0}}, s\right)<\varepsilon
$$

Hence, $\left\{s_{v}\right\}$ is a Cauchy sequence in closed subset $K$ of $\Sigma$; therefore, it converges in $K$ such that

$$
\lim _{v \longrightarrow \infty} s_{v}=q \text { for } q \in K .
$$

As we have $\lim _{v \longrightarrow \infty} \sigma\left(s_{v}, F(Y)\right)=0 \Rightarrow \sigma(q, F(Y))=0$, since $F(Y)$ is closed so $q \in F(Y)$.

Now, we prove that newly proposed iteration scheme (1) is faster than Thakur New [25] for a mapping defined in (2) in hyperbolic metric space.

Theorem 19. Let $(\Sigma, \sigma, \preccurlyeq)$ be a P.O H.M.S. Let $K$ be a nonempty convex closed bounded subset of $\Sigma$, and $Y$ be a mapping satisfying (30) with $F(Y) \neq \vartheta$. Let $\left\{s_{v}\right\}$ be defined by (28), and $\left\{u_{v}\right\}$ defined in [25], then $\left\{s_{v}\right\}$ converges faster than $\left\{u_{v}\right\}$.

Proof. Let $s \in F(Y) \Rightarrow Y^{v} s=s$. Now, using (28) and (30), we have

$$
\begin{aligned}
\sigma\left(\eta_{v}, s\right) & \leq\left(1-\gamma_{v}\right) \sigma\left(s_{v}, s\right)+\gamma_{v}\left[a \sigma\left(s_{v}, s\right)+L \sigma\left(s, Y^{v} s\right)\right] \\
& =\left(1-\gamma_{v}(1-a)\right) \sigma\left(s_{v}, s\right) .
\end{aligned}
$$

Again, using (28), (30), and (58), we have

$$
\sigma\left(\theta_{v}, s\right) \leq\left(1-\beta_{v}(1-a)\right)\left(1-\gamma_{v}(1-a)\right) \sigma\left(s_{v}, s\right)
$$

Further, consider

$$
\sigma\left(s_{v+1}, s\right)=\sigma\left(Y^{v}\left(\left(1-\alpha_{v}\right) Y^{v} \eta_{v} \oplus \alpha_{v} Y^{v} \theta_{v}\right), s\right)
$$

Now, using (30) and then (28), we get

$$
\sigma\left(s_{v+1}, s\right) \leq a\left[a\left(1-\gamma_{v}(1-a)\right)\left(1-(1-a) \alpha_{v} \beta_{v}\right)\right] \sigma\left(s_{v}, s\right) .
$$

Let

$$
K_{v}=a^{v}\left[a[(1-\gamma(1-a))(1-(1-a) \alpha \beta)]^{v}\right] \sigma\left(s_{1}, s\right),
$$

and $\sigma_{v}=a^{v}[(1-\gamma(1-a))(1-(1-a) \alpha \beta)]^{v} \sigma\left(u_{1}, s\right) \quad$ calculated in Theorem 3.1 of Thakur New [25]. Then,

$$
\begin{aligned}
\frac{K_{v}}{\sigma_{v}} & =\frac{a^{v}\left[a[(1-\gamma(1-a))(1-(1-a) \alpha \beta)]^{v}\right] \sigma\left(s_{1}, s\right)}{a^{v}[(1-\gamma(1-a))(1-(1-a) \alpha \beta)]^{v} \sigma\left(u_{1}, s\right)} \\
& =\frac{a[(1-\gamma(1-a))(1-(1-a) \alpha \beta)]^{v} \sigma\left(s_{1}, s\right)}{[(1-\gamma(1-a))(1-(1-a) \alpha \beta)]^{v} \sigma\left(u_{1}, s\right)} \longrightarrow 0 \text { as } v \longrightarrow \infty
\end{aligned}
$$

Hence, $\left\{s_{v}\right\}$ converges faster than $\left\{u_{v}\right\}$.

Now, we will prove the stability result for this we have the following definition by [30].

Definition 20. Let $\left\{p_{v}\right\}_{v=0}^{\infty} \subset K$ be any arbitrary sequence, then iteration sequence $s_{v+1}$ converging to unique fixed point $s$ is said to be $Y$-stable if for $\varepsilon_{v}=\sigma\left(p_{v}, s_{v+1}\right) v \geq 0$, we have

$$
\lim _{v \longrightarrow \infty} \varepsilon_{v}=0 \Leftrightarrow \lim _{v \longrightarrow \infty} p_{v}=s .
$$

Theorem 21. Let $\left(\Sigma, \sigma^{\prime}\right)$ be a P.O H.M.S. Let $K$ be a nonempty convex closed bounded subset of $\Sigma$, and $Y$ be a mapping satisfying (2) with $F(Y) \neq \vartheta$. Let $\left\{s_{v}\right\}$ be defined by (1), satisfying $\sum \alpha_{v}=\infty$, then the iteration (1) is $Y$-stable.

Proof. Let $\left\{p_{v}\right\}_{v=0}^{\infty} \subset K$ be any arbitrary sequence, the sequence defined by (28) converging to unique fixed point $s$, and

$$
\varepsilon_{v}=\sigma\left(p_{v}, s_{v+1}\right)
$$

We have to prove that

$$
\lim _{v \longrightarrow \infty} \varepsilon_{v}=0 \Leftrightarrow \lim _{v \longrightarrow \infty} p_{v}=s
$$


TABle 1: The convergence behavior of Mann, Ishikawa, Agarwal, and Noor iterations with new iteration for the parameters $\alpha=0: 6$, $\beta=0: 3$, and $\gamma=0: 5$, with the initial values $x_{0}=0, y_{0}=3$, and tolerance $=10^{-6}$.

\begin{tabular}{|c|c|c|c|c|c|}
\hline Steps & Mann & Ishikawa & Agarwal & Noor & New \\
\hline 0 & $(0,3)$ & $(0,3)$ & $(0,3)$ & $(0,3)$ & $(0,3)$ \\
\hline 1 & $(0,2.244506)$ & $(0,2.013105)$ & $(0,1.509443)$ & $(0,1.916515)$ & $(0,0.311990)$ \\
\hline 2 & $(0,1.519333)$ & $(0,1.196547)$ & $(0,0.479098)$ & $(0,1.097796)$ & $(0,0.011353)$ \\
\hline 3 & $(0,0.949002)$ & $(0,0.663545)$ & $(0,0.116180)$ & $(0,0.594503)$ & $(0,0.000384)$ \\
\hline 4 & $(0,0.561764)$ & $(0,0.355178)$ & $(0,0.025817)$ & $(0,0.313100)$ & $(0,0.000012)$ \\
\hline 5 & $(0,0.321995)$ & $(0,0.186769)$ & $(0,0.005616)$ & $(0,0.162620)$ & $(0,0.000000)$ \\
\hline 6 & $(0,0.181202)$ & $(0,0.097333)$ & $(0,0.001216)$ & $(0,0.083873)$ & $(0,0.000000)$ \\
\hline 7 & $(0,0.100930)$ & $(0,0.050492)$ & $(0,0.000263)$ & $(0,0.043105)$ & $(0,0.000000)$ \\
\hline 8 & $(0,0.055900)$ & $(0,0.026132)$ & $(0,0.000056)$ & $(0,0.022113)$ & $(0,0.000000)$ \\
\hline 9 & $(0,0.030863)$ & $(0,0.013508)$ & $(0,0.000012)$ & $(0,0.011333)$ & $(0,0.000000)$ \\
\hline 10 & $(0,0.017010)$ & $(0,0.006978)$ & $(0,0.000002)$ & $(0,0.005806)$ & $(0,0.000000)$ \\
\hline 11 & $(0,0.009366)$ & $(0,0.003603)$ & $(0,0.000000)$ & $(0,0.002973)$ & $(0,0.000000)$ \\
\hline 12 & $(0,0.005155)$ & $(0,0.001860)$ & $(0,0.000000)$ & $(0,0.001522)$ & $(0,0.000000)$ \\
\hline 13 & $(0,0.002836)$ & $(0,0.000960)$ & $(0,0.000000)$ & $(0,0.000779)$ & $(0,0.000000)$ \\
\hline 14 & $(0,0.001560)$ & $(0,0.000495)$ & $(0,0.000000)$ & $(0,0.000399)$ & $(0,0.000000)$ \\
\hline 15 & $(0,0.000858)$ & $(0,0.000256)$ & $(0,0.000000)$ & $(0,0.000204)$ & $(0,0.000000)$ \\
\hline 16 & $(0,0.000472)$ & $(0,0.000132)$ & $(0,0.000000)$ & $(0,0.000104)$ & $(0,0.000000)$ \\
\hline 17 & $(0,0.000259)$ & $(0,0.000068)$ & $(0,0.000000)$ & $(0,0.000053)$ & $(0,0.000000)$ \\
\hline 18 & $(0,0.000142)$ & $(0,0.000035)$ & $(0,0.000000)$ & $(0,0.000027)$ & $(0,0.000000)$ \\
\hline 19 & $(0,0.000078)$ & $(0,0.000018)$ & $(0,0.000000)$ & $(0,0.000014)$ & $(0,0.000000)$ \\
\hline 20 & $(0,0.000043)$ & $(0,0.000009)$ & $(0,0.000000)$ & $(0,0.000007)$ & $(0,0.000000)$ \\
\hline 21 & $(0,0.000023)$ & $(0,0.000004)$ & $(0,0.000000)$ & $(0,0.000003)$ & $(0,0.000000)$ \\
\hline 22 & $(0,0.000013)$ & $(0,0.000002)$ & $(0,0.000000)$ & $(0,0.000001)$ & $(0,0.000000)$ \\
\hline 23 & $(0,0.000007)$ & $(0,0.000001)$ & $(0,0.000000)$ & $(0,0.000000)$ & $(0,0.000000)$ \\
\hline 24 & $(0,0.000003)$ & $(0,0.000000)$ & $(0,0.000000)$ & $(0,0.000000)$ & $(0,0.000000)$ \\
\hline 25 & $(0,0.000002)$ & $(0,0.000000)$ & $(0,0.000000)$ & $(0,0.000000)$ & $(0,0.000000)$ \\
\hline 26 & $(0,0.000001)$ & $(0,0.000000)$ & $(0,0.000000)$ & $(0,0.000000)$ & $(0,0.000000)$ \\
\hline 27 & $(0,0.000000)$ & $(0,0.000000)$ & $(0,0.000000)$ & $(0,0.000000)$ & $(0,0.000000)$ \\
\hline
\end{tabular}

Let $\lim _{v \longrightarrow \infty} \varepsilon_{v}=0$, then

$$
\begin{aligned}
\sigma\left(p_{v}, s\right) \leq & \sigma\left(p_{v}, s_{v+1}\right)+\sigma\left(s_{v+1}, s\right) \\
= & \varepsilon_{v}+\sigma\left(Y^{v}\left(\left(1-\alpha_{v}\right) Y^{v} s_{v} \oplus \alpha_{v} Y^{v} \theta_{v}\right), s\right) \\
\leq & \varepsilon_{v}+a \sigma\left(\left(1-\alpha_{v}\right) Y^{v} \eta_{v} \oplus \alpha_{v} Y^{v} \theta_{v}, s\right) \\
& +L \sigma\left(s, Y^{v} s\right)=\varepsilon_{v}+a \sigma\left(\left(1-\alpha_{v}\right)\right. \\
& \left.\cdot Y^{v} \eta_{v} \oplus \alpha_{v} Y^{v} \theta_{v}, s\right) \leq \varepsilon_{v}+a\left[a \left(1-\gamma_{v}\right.\right. \\
& \left.\cdot(1-a))\left(1-(1-a) \alpha_{v} \beta_{v}\right)\right] \sigma\left(s_{v}, s\right),
\end{aligned}
$$$$
0 \leq a\left[a\left(1-\gamma_{v}(1-a)\right)\left(1-(1-a) \alpha_{v} \beta_{v}\right)\right]<1, \quad \text { applying }
$$$$
\lim _{v \longrightarrow \infty} \text {, we get }
$$

$$
\lim _{v \longrightarrow \infty} \sigma\left(p_{v}, s\right)=0 \Rightarrow \lim _{v \longrightarrow \infty} p_{v}=s
$$

(1)

applying

\begin{tabular}{lcccc}
\hline Steps & Abbas & An accelerated & Thakur & New \\
\hline 0 & $(0,3)$ & $(0,3)$ & $(0,3)$ & $(0,3)$ \\
1 & $(0,0.917554)$ & $(0,0.890744)$ & $(0,0.969760)$ & $(0,0.311990)$ \\
2 & $(0,0.133217)$ & $(0,0.126269)$ & $(0,0.161770)$ & $(0,0.011353)$ \\
3 & $(0,0.015917)$ & $(0,0.015024)$ & $(0,0.022578)$ & $(0,0.000384)$ \\
4 & $(0,0.001852)$ & $(0,0.001747)$ & $(0,0.003065)$ & $(0,0.000012)$ \\
5 & $(0,0.000214)$ & $(0,0.000202)$ & $(0,0.000414)$ & $(0,0.000000)$ \\
6 & $(0,0.000024)$ & $(0,0.000023)$ & $(0,0.000056)$ & $(0,0.000000)$ \\
7 & $(0,0.000002)$ & $(0,0.000002)$ & $(0,0.000007)$ & $(0,0.000000)$ \\
8 & $(0,0.000000)$ & $(0,0.000000)$ & $(0,0.000001)$ & $(0,0.000000)$ \\
9 & $(0,0.000000)$ & $(0,0.000000)$ & $(0,0.000000)$ & $(0,0.000000)$ \\
\hline
\end{tabular}

8

西

TABle 2: The convergence of Abbas, Thakur, and accelerated iteration with new iteration for the same initial values, parameters, and tolerance $10^{-6}$.

Conversely, let $\lim _{v \longrightarrow \infty} p_{v}=s$, we have 


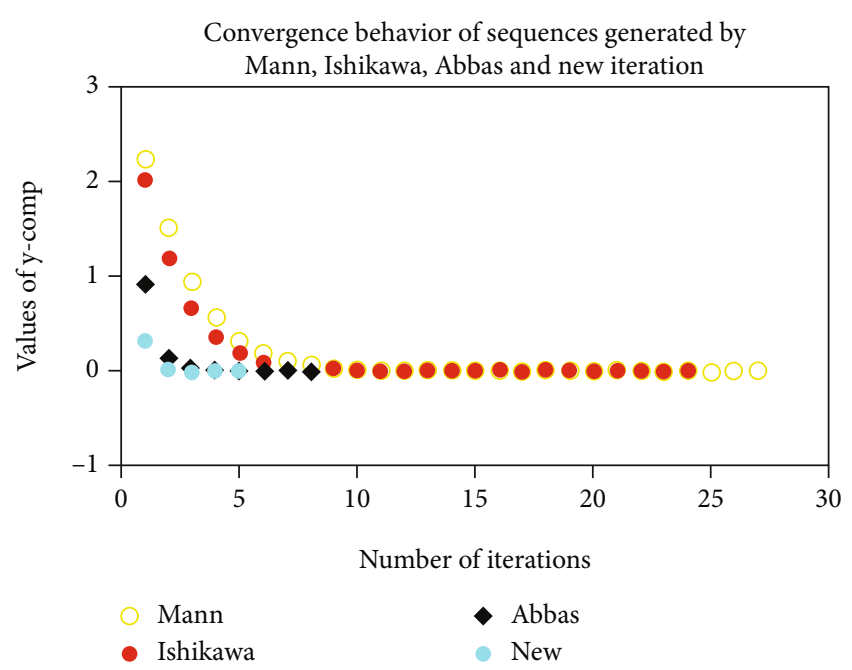

Figure 1: Convergence behavior of Mann, Ishikawa, and Abbas with new iteration for the initial values $(0,3)$ with parameters $\alpha=$ $0.6, \beta=0.3, \gamma=0.5$.

$$
\begin{aligned}
\varepsilon_{v}= & \sigma\left(p_{v}, s_{v+1}\right) \leq \sigma\left(p_{v}, s\right)+\sigma\left(s_{v+1}, s\right) \leq \sigma\left(p_{v}, s\right) \\
& +a\left[a\left(1-\gamma_{v}(1-a)\right)\left(1-(1-a) \alpha_{v} \beta_{v}\right)\right] \sigma\left(s_{v}, s\right) \\
& \Rightarrow \lim _{v \longrightarrow \infty} \varepsilon_{v}=0 .
\end{aligned}
$$

Hence, it is $Y$-stable.

We have a nontrivial example for M.T.A.N.M, and fixed point is numerically approximated by using MATLAB.

Example 3. Let $\Sigma=\mathbb{R}^{2}$ be a hyperbolic space. Define a relation as

$$
\left(r_{1}, t_{1}\right)^{\prime}\left(r_{2}, t_{2}\right) \Leftrightarrow r_{1} \leq r_{2} \text { and } t_{1} \leq t_{2} \text {. }
$$

Let $\sigma: \Sigma \times \Sigma \longrightarrow \mathbb{R}$ be defined as

$\sigma(r, t)=\left|r_{1}-r_{2}\right|+\left|r_{1} t_{1}-r_{2} t_{2}\right|$ where $r=\left(r_{1}, t_{1}\right), y=\left(r_{2}, t_{2}\right)$.

Let $K=[0,3] \times[0,3] \subset \Sigma$ and $Y: K \longrightarrow K$ be a mapping defined by

$$
Y(r, t)=\left\{\left(\frac{(1-\cos r)}{2}, \frac{\exp (t / 2)-1}{2}\right) ;(r, t) \in K .\right.
$$

As $(0,0)$ is the fixed point of $Y$, we have to show that $Y$ is monotone for this consider

$$
\left(r_{1}, t_{1}\right)^{\prime}\left(r_{2}, t_{2}\right) \Leftrightarrow r_{1} \leq r_{2} \text { and } t_{1} \leq t_{2},
$$

then

$$
\frac{1-\cos r_{1}}{2} \leq \frac{1-\cos r_{2}}{2} \text { and } \frac{\exp \left(t_{1} / 2\right)-1}{2} \leq \frac{\exp \left(t_{2} / 2\right)-1}{2} \text {, }
$$

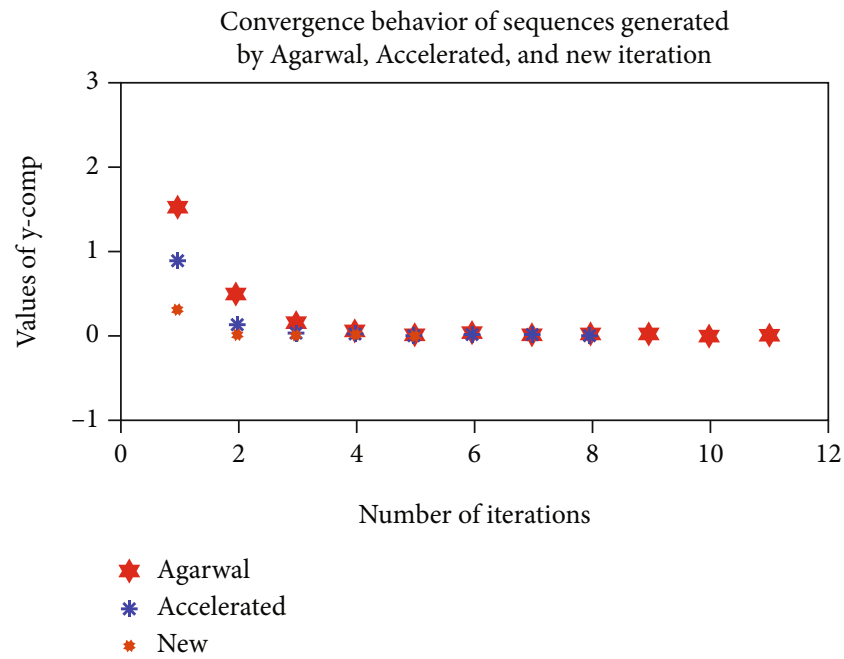

Figure 2: Convergence behavior of Agarwal, accelerated with new iteration for the initial values $(0,3)$ with parameters $\alpha=0.6, \beta=0.3$, $\gamma=0.5$.

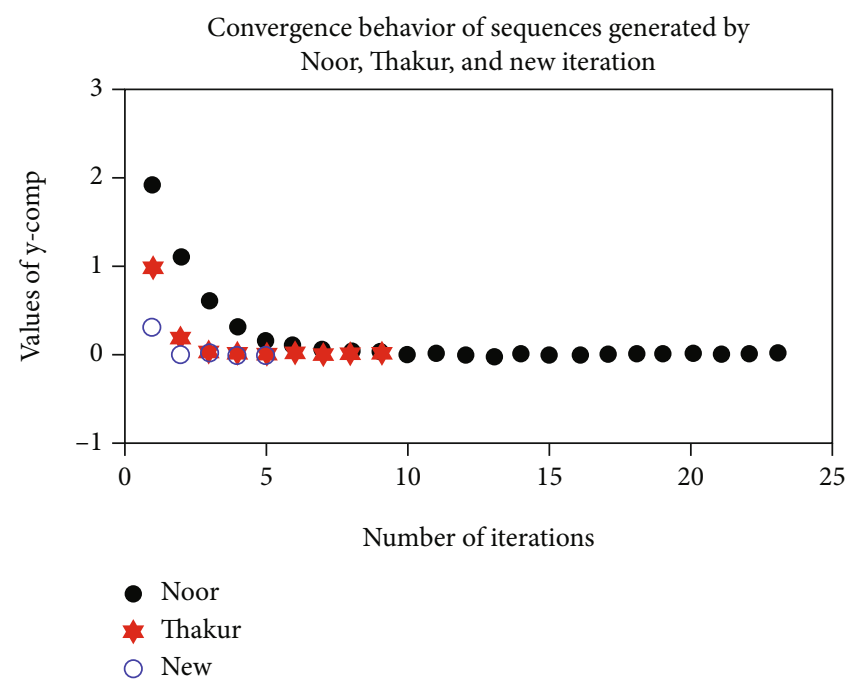

FIgURE 3: Convergence behavior of Noor and Thakur with new iteration for the initial values $(0,3)$ with parameters $\alpha=0.6, \beta=0.3$, $\gamma=0.5$.

that is

$$
Y\left(r_{1}, t_{1}\right)^{\prime} Y\left(r_{2}, t_{2}\right)
$$

Next,

$$
\begin{aligned}
Y^{v}\left(r_{1}, t_{1}\right)= & (0,0), Y^{v}\left(r_{2}, t_{2}\right)=(0,0), \text { for large } v, \\
& \Rightarrow \sigma\left(Y^{v}\left(r_{1}, t_{1}\right), Y^{v}\left(r_{2}, t_{2}\right)\right) \\
= & 0 \leq \sigma\left(\left(r_{1}, t_{1}\right),\left(r_{2}, t_{2}\right)\right) .
\end{aligned}
$$

Hence, $Y$ is M.T.A.N.M with $\mu_{v}=\xi_{v}=0$.

The rate of convergence of Mann, Ishikawa, Agarwal, Noor, Abbas, Thakur, and accelerated and new iterations 
TABLE 3: Influence of parameters and initial values by setting stopping parameter at $10^{-15}$.

\begin{tabular}{|c|c|c|c|c|c|c|c|c|}
\hline \multicolumn{9}{|c|}{ For $\alpha=0.7, \beta=\gamma=0.1$ with $\left(x_{0}, y_{0}\right)=(0,3)$} \\
\hline Iteration process & Mann & Ishikawa & Agarwal & Noor & Abbas & An accelerated & Thakur & New \\
\hline Number of iterations & 50 & 48 & 26 & 48 & 22 & 22 & 25 & 13 \\
\hline \multicolumn{9}{|c|}{ For $\alpha=0.2=\beta, \gamma=0.3$ with $\left(x_{0}, y_{0}\right)=(0,2.5)$} \\
\hline Iteration process & Mann & Ishikawa & Agarwal & Noor & Abbas & An accelerated & Thakur & New \\
\hline Number of iterations & 221 & 202 & 26 & 203 & 15 & 15 & 22 & 12 \\
\hline \multicolumn{9}{|c|}{ For $\alpha=0.3=\beta=0.7, \gamma=0.2$ with $\left(x_{0}, y_{0}\right)=(0,2)$} \\
\hline Iteration process & Mann & Ishikawa & Agarwal & Noor & Abbas & An accelerated & Thakur & New \\
\hline Number of iterations & 140 & 116 & 24 & 115 & 16 & 16 & 21 & 12 \\
\hline \multicolumn{9}{|c|}{ For $\alpha=0.5=\beta=0.9, \gamma=0.2$ with $\left(x_{0}, y_{0}\right)=(0,1)$} \\
\hline Iteration process & Mann & Ishikawa & Agarwal & Noor & Abbas & An accelerated & Thakur & New \\
\hline Number of iterations & 74 & 57 & 20 & 56 & 18 & 18 & 18 & 11 \\
\hline \multicolumn{9}{|c|}{ For $\alpha=0.3=\beta=0.9, \gamma=0.5$ with $\left(x_{0}, y_{0}\right)=(0,1.5)$} \\
\hline Iteration process & Mann & Ishikawa & Agarwal & Noor & Abbas & An accelerated & Thakur & New \\
\hline Number of iterations & 138 & 109 & 23 & 106 & 14 & 14 & 17 & 11 \\
\hline
\end{tabular}

for the mapping defined in Example 2 is given below. Table 1 shows the convergence behavior of Mann, Ishikawa, Agarwal, and Noor iterations with new iteration for the parameters $\alpha=0.6, \beta=0.3$, and $\gamma=0.5$, with the initial values $x_{0}=0, y_{0}=3$, and tolerance $=10^{-6}$. New iteration requires less number of iterations for convergence.

Table 2 shows the convergence of Abbas, Thakur, and accelerated iteration with new iteration for the same initial values, parameters, and tolerance.

The following figures show the convergence behavior of different iterations with new iteration in Figures 1-3.

All iterations converges to $(x, y)=(0,0)$. Comparison shows that new iteration requires the least number of iterations for convergence. Table 3 shows that different parameters have an effect on iterations and by changing the initial values, new iteration not only converges faster but also stable than other iterations

\section{Conclusions}

In the present article, the concept of monotone asymptotically nonexpansive mapping has been generalized to monotone total asymptotically nonexpansive mapping in the framework of hyperbolic space. New iteration has been introduced to approximate the fixed point for that mapping. We proved the existence of fixed point, faster convergence, and stability results for new iteration. We also constructed a nontrivial example to approximate the fixed point numerically and compare the convergence result of new iteration with some well-known iterations by using MATLAB.

By relaxing the condition of monotonicity, we can also achieve some similar results presented in recent articles [31, 32] by using the proposed iteration.

\section{Data Availability}

No data were used to support this study.

\section{Conflicts of Interest}

The authors have no conflict of interests regarding the publication of this paper.

\section{Acknowledgments}

The research was supported by the National Natural Science Foundation of China (Grant Nos. 11971142, 11871202, $61673169,11701176,11626101$, and 11601485) and the Taif University Researchers Supporting Project number (TURSP-2020/77), Taif University, Taif, Saudi Arabia.

\section{References}

[1] S. Reich and I. Shafrir, "Nonexpansive iterations in hyperbolic spaces," Nonlinear Analysis: Theory, Methods \& Applications, vol. 15, no. 6, pp. 537-558, 1990.

[2] W. Takahashi, "A convexity in metric space and nonexpansive mappings, I.," Kodai Mathematical Journal, vol. 22, no. 2, pp. 142-149, 1970.

[3] B. Ali, "Convergence theorems for finite families of total asymptotically nonexpansive mappings in hyperbolic spaces," Fixed Point Theory and Applications, vol. 2016, no. 1, Article ID 24, 2016.

[4] A. Pansuwan and W. Sintunavarat, "A new iterative scheme for numerical reckoning fixed points of total asymptotically nonexpansive mappings," Fixed Point Theory and Applications, vol. 2016, no. 1, Article ID 83, 2016.

[5] M. Bachar and M. A. Khamsi, "Fixed points of monotone mappings and application to integral equations," Fixed Point Theory and Applications, vol. 2015, no. 1, Article ID 110, 2015.

[6] B. A. Bin Dehaish and M. A. Khamsi, "Browder and Göhde fixed point theorem for monotone nonexpansive mappings," Fixed Point Theory and Applications, vol. 2016, no. 1, Article ID 20, 2016.

[7] F. E. Browder, "Nonexpansive nonlinear operators in a Banach space," Proceedings of the National Academy of Sciences of the United States of America, vol. 54, no. 4, pp. 1041-1044, 1965. 
[8] D. Göhde, "Zum prinzip der kontraktiven abbildung," Mathematische Nachrichten, vol. 30, no. 3-4, pp. 251-258, 1965.

[9] M. Alfuraidan and M. Khamsi, "A fixed point theorem for monotone asymptotically nonexpansive mappings," Proceedings of the American Mathematical Society, vol. 146, no. 6, pp. 2451-2456, 2018.

[10] K. Goebel and W. A. Kirk, "A fixed point theorem for asymptotically nonexpansive mappings," Proceedings of the American Mathematical Society, vol. 35, no. 1, pp. 171-174, 1972.

[11] M. A. A. Khan, "Convergence analysis of a multi-step iteration for a finite family of asymptotically quasi-nonexpansive mappings," Journal of Inequalities and Applications, vol. 2013, no. 1, Article ID 423, 2013.

[12] H. Fukhar-ud-din and M. A. A. Khan, "Convergence analysis of a general iteration schema of nonlinear mappings in hyperbolic spaces," Fixed Point Theory and Applications, vol. 2013, no. 1, Article ID 238, 2013.

[13] W. Liu, Y. Wang, and S. Y. Cho, "The split equality fixed-point problem for totally quasi-asymptotically strictly pseudocontractive mappings without prior knowledge of operator norms," Journal of Nonlinear Functional Analysis, vol. 2019, no. 1, article 43, 2019.

[14] B. Tan and S. Li, "Strong convergence of inertial Mann algorithms for solving hierarchical fixed point problems," Journal of Nonlinear and Variational Analysis, vol. 4, no. 3, pp. 337355, 2020.

[15] Y. Alber, C. E. Chidume, and H. Zegeye, "Approximating fixed points of total asymptotically nonexpansive mappings," Fixed Point Theory and Applications, vol. 2006, no. 1, Article ID 10673, 2006.

[16] E. U. Ofoedu and A. C. Nnubia, "Approximation of minimum-norm fixed point of total asymptotically nonexpansive mapping," Afrika Matematika, vol. 26, no. 5-6, pp. 699715, 2015.

[17] S. Banach, "Sur les opérations dans les ensembles abstraits et leur application aux équations intégrales," Fundamenta Mathematicae, vol. 3, no. 1, pp. 133-181, 1922.

[18] W. R. Mann, "Mean value methods in iteration," Proceedings of the American Mathematical Society, vol. 4, no. 3, pp. 506510, 1953.

[19] S. Ishikawa, "Fixed points by a new iteration method," Proceedings of the American Mathematical Society, vol. 44, no. 1, pp. 147-150, 1974.

[20] R. P. Agarwal, D. O. Regan, and D. R. Sahu, "Iterative construction of fixed points of nearly asymptotically nonexpansive mappings," Journal of Nonlinear and Convex Analysis, vol. 8, no. 1, p. $61,2007$.

[21] M. A. Noor, "New approximation schemes for general variational inequalities," Journal of Mathematical Analysis and Applications, vol. 251, no. 1, pp. 217-229, 2000.

[22] M. Abbas and T. Nazir, "A new faster iteration process applied to constrained minimization and feasibility problems," Matematicki Vesnik, vol. 66, no. 2, pp. 223-234, 2014.

[23] V. Karakaya, N. E. H. Bouzara, K. Dogan, and Y. Atalan, “On different results for a new two-step iteration method under weak-contraction mappings in Banach spaces," 2015, https:// arxiv.org/abs/1507.00200.

[24] Y. A. Chen and D. J. Wen, "Convergence analysis of an accelerated iteration for monotone generalized $\alpha$-nonexpansive mappings with a partial order," Journal of Function Spaces, vol. 2019, Article ID 2789819, 8 pages, 2019.
[25] B. S. Thakur, D. Thakur, and M. Postolache, "A new iteration scheme for approximating fixed points of nonexpansive mappings," Filomat, vol. 30, no. 10, pp. 2711-2720, 2016.

[26] R. Pandey, R. Pant, and A. al-Rawashdeh, "Fixed point results for a class of monotone nonexpansive type mappings in hyperbolic spaces," Journal of Function Spaces, vol. 2018, Article ID 5850181, 11 pages, 2018.

[27] T. C. Lim, "Remarks on some fixed point theorems," Proceedings of the American Mathematical Society, vol. 60, no. 1, pp. 179-182, 1976.

[28] V. Berinde, "A convergence theorem for some mean value fixed point iteration procedures," Demonstratio Mathematica, vol. 38, no. 1, pp. 177-184, 2005.

[29] V. Berinde, "Picard iteration converges faster than Mann iteration for a class of quasi-contractive operators," Fixed Point Theory and Applications, vol. 2004, no. 2, p. 105, 2004.

[30] A. M. Harder, Fixed Point Theory and Stability Results for Fixed Point Iteration Procedure, 1988.

[31] I. Uddin, S. Khatoon, N. Mlaiki, and T. Abdeljawad, “A modified iteration for total asymptotically nonexpansive mappings in Hadamard spaces," AIMS Mathematics, vol. 6, no. 5, pp. 4758-4770, 2021.

[32] N. Hussain, K. Ullah, and M. Arshad, "Fixed point approximation of Suzuki generalized nonexpansive mappings via new faster iteration process," Journal of Nonlinear and Convex Analysis, vol. 19, no. 8, pp. 1383-1393, 2018. 